\title{
Trust your gut - metformin and diabetes
}

The mechanism of action of metformin - an anti-diabetic drug that is widely used to treat type 2 diabetes mellitus (T2DM) - is poorly defined, and is generally thought to suppress hepatic gluconeogenesis. However, emerging evidence implicates the gut microbiota as a major determinant of metformin action. Adding impetus to this hypothesis, a study published in Nature Medicine reports that metformin induces marked alterations in the gut microbiota of individuals with treatment-naive T2DM, and that this effect mediates the glucose-lowering effects of metformin. To investigate the effect of metformin on the composition of the gut microbiota, investigators carried out a double-blind study; participants with treatment-naive T2DM were randomly assigned to receive either placebo $(n=18)$ or metformin $(1,700 \mathrm{mg}$ per day; $n=22$ ) for 4 months. 6 months after the randomized study, a subset of the placebo group was switched to treatment with

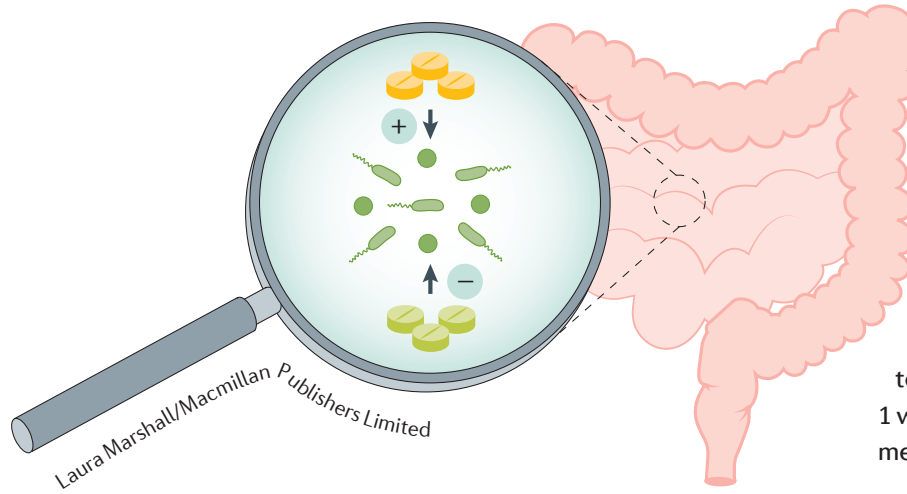

metformin for 6 months ( 850 or $1,700 \mathrm{mg}$ per day; $n=13$ ), and this cohort was used for validation. Stool samples were collected from participants of both studies and were used for analysis.

Whole-genome shotgun sequencing of faecal samples demonstrated that treatment with metformin induced major alterations in the relative abundance of $>80$ bacterial strains compared with placebo. Specifically, metformin increased and decreased the abundance of bacteria in the genera Escherichia and Intestinibacter, respectively. Although in vitro studies showed that metformin-dependent increases in the abundance of Escherichia coli were not due to increased growth, investigators found that metformin promoted the growth of Bifidobacterium adolescentis both in vitro and in faecal samples. Interestingly, faecal transfer to germ-free mice improved glucose tolerance in recipients of samples from two of three patients treated with metformin, illustrating that metformin-altered gut microbiota can promote glucose-lowering effects.

To model metformin-dependent modulation of the composition of the gut microbiota, investigators used an in vitro 'gut simulator' cultured faecal samples obtained before metformin treatment from two participants were exposed to a constant flow of metformin for 1 week, after which metagenomic and metatranscriptomic analyses were carried out. "This methodology allowed us to investigate the direct effects of metformin on the microbiome rather than secondary effects mediated by the host," explains author Fredrik Bäckhed. Although no changes in the abundance of $E$. coli or B. adolescentis were seen, metformin increased the abundance of 24 and four bacterial strains in the two experiments, respectively. Akkermansia muciniphila was the only taxa increased by metformin in both samples and had the largest increase in abundance with metformin. Transcriptional analysis in A. muciniphila demonstrated that the majority of metformin-regulated genes encoded metalloproteins or metal transporters, which might indicate a functional link between metformin and these microbial proteins.

Overall, the results infer a new mechanism of metformin action, whereby metformin-dependent modulation of the gut microbiota might mediate some of the anti-diabetic effects of this drug. "The next step is to study the mechanisms by which metformin-altered microbiota lower blood glucose levels," concludes author José Manuel Fernàndez-Real.

Conor A. Bradley This article originally appeared in Nat. Rev. Gastroenterol. Hepatol. (http://dx.doi.org/10.1038/ nrgastro.2017.73)

ORIGINAL ARTICLE Wu, H. et al. Metformin alters the gut microbiome of individuals with treatment-naive type 2 diabetes, contributing to the therapeutic effects of the drug. Nat. Med. http://dx.doi.org/10.1038/nm.4345 (2017) 